\title{
Leaf expansion in Arabidopsis is controlled by a TCP-NGA regulatory module likely conserved in distantly related species
}

\author{
Patricia Ballester, Marisa Navarrete-Gómez , Pilar Carbonero , Luis Oñate-Sánchez and Cristina \\ Ferrándiz
}

The NGATHA (NGA) clade of transcription factors (TFs) forms a small sub-family of four members in Arabidopsis thaliana. NGA genes act redundantly to direct the development of apical tissues in the gynoecium, where they have been shown to be essential for style and stigma specification. In addition, NGA genes have a more general role in controlling lateral organ growth. The four NGA genes in Arabidopsis are expressed in very similar domains, although little is known about the nature of their putative regulators. Here, we have identified a conserved region within the four NGA promoters that we have used as a bait to screen a yeast library, aiming to identify such NGA regu-lators. Three members of the TCP family of TFs, named after the founding factors TEOSINTE BRANCHED 1, CYCLOIDEA and PROLIFERATING CELL FACTOR 1 AND 2), were recovered from this screening, of which two [TCP2 and TCP3, members of the CINCINNATA (CIN) family of TCP genes (CIN-TCP) subclade] were shown to activate the NGA3 promoter in planta. We pro-vide evidence that support that CIN-TCP genes are true regulators of NGA gene expression, and that part of the CIN-TCP role in leaf development is mediated by NGA upregulation. Moreover, we have found that this TCP-NGA regulatory interaction is likely conserved in angiosperms, including impor-tant crop species, for which the regulation of leaf development is a target for biotechnological improvement.

\section{Introduction}

NGATHA (NGA) genes have a key role in the specification of style and stigma in the gynoecium, which has been shown to be conserved in distantly related species within the eudicot clade of Angiosperms (Alvarez et al. 2009, Trigueros et al. 2009, Fourquin and Ferran-diz 2014). NGA genes were first characterized in Arabidopsis thaliana, where they form a small subfamily of four highly related $B 3$ transcription factors (TFs), NGA1, NGA2, NGA3 and NGA4 (Araki and Komeda 1993, Bleecker 1998, Alvarez et al. 2009, Trigueros et al. 2009). NGA factors group together with other members of the RELATED TO AP2 AND VP1 (RAV) clade in this family and are characterized by the presence of a sin-gle B3 DNA-binding domain and three C-terminal motifs known as NGA-I motif, RAVrepressive domain and NGA-II motif (Fourquin and Ferrandiz 2014). The four

Abbreviations - BAC, Bacterial Artificial Chromosome; CDS, coding sequence; CIN, CINCINNATA; Col-0, columbia-0; CUC, CUP-SHAPED COTYLEDON; GUS, $\beta$-GLUCURONIDASE; LA, LANCEOLATE; LUC, LUCIFERASE; miRNA, miCroRNA; NGA, NGATHA; NGAL, NGATHA-LIKE; ORF, Open Reading Frame; PCR, polymerase chain reaction; RAV, RELATED TO AP2 AND VP1; REN, Renilla; TF, transcription factor; UTR, untranslated region; $Y 1 H$, yeast one-hybrid. 
NGA genes in Arabidopsis are functionally redundant. Loss of NGA function results in the absence of the apical tissues within the pistil, which in quadruple nga mutants ends as an open cylinder with distal protrusions of valve-like tissue. In addition to this role in gynoecium development, NGA genes are important general regulators of lateral organ growth. The leaves of Arabidopsis quadruple nga mutants are wider and shorter than wild type, and these phenotypes are also observed in other floral organs, specially sepals and petals (Fourquin and Ferrandiz 2014). Conversely, NGA overexpression produces small narrow leaves and petals (Alvarez et al. 2009, Kwon et al. 2009, Trigueros et al. 2009).

NGA genes have been related to auxin signaling (Trigueros et al. 2009, Martinez-Fernandez et al. 2014). Thus, it has been shown that NGA factors positively regulate local auxin synthesis and also that NGA altered expression leads to differential expression of several Auxin Response Factors and auxin transporters (Martinez-Fernandez et al. 2014). Defective auxin signaling has been associated with altered leaf morphology and carpel patterning defects, so, at least partly, the phenotypes observed in nga mutants or overexpressors could be related to altered auxin pathways (Ostergaard 2009, Sundberg and Ferrándiz 2009, Sundberg and Østergaard 2009, Koenig and Sinha 2010). However, auxin appears to have only little influence on NGA gene expression (Trigueros et al. 2009), suggesting that other upstream regulators remain to be identified.

The four NGA genes in Arabidopsis share very similar expression patterns. In leaf primordia, expression is associated with distal marginal tissues in very young primordia, restricted later to the tips of cotyledons and leaves and to leaf hydathodes. In floral organs, NGA expression is mainly detected in the distal part of growing primordia, disappearing in expanded sepals and petals. In the gynoecium, NGA accumulates in the apical end of the growing gynoecial tube, restricting later to the incipient style and stigma and the placental tissues (Alvarez et al. 2009, Trigueros et al. 2009). The highly similar expression patterns of all four NGA genes strongly suggest that they may share common regulators. In this work, we have identified a conserved region among the four NGA promoters and hypothesized that it was likely bound by these putative regulators. Using a yeast one-hybrid $(\mathrm{Y} 1 \mathrm{H})$ strategy followed by molecular and genetic analyses, we have shown that some miR319-regulated TCP factors are activators of NGA expression and that NGA factors partly mediate TCP roles in leaf morphogenesis. Moreover, we have observed that this TCP-NGA regulatory module appears to be conserved in distantly related species, including crops such as tomato, rice or common bean.

\section{Materials and methods}

\section{Plant material and growth conditions}

Arabidopsis thaliana plants were grown in cabinets at $21^{\circ} \mathrm{C}$ under long day $(16 \mathrm{~h}$ light) or short day $(8 \mathrm{~h}$ light) conditions, illuminated by cool-white fluorescent lamps $\left(150 \mu \mathrm{mol} \mathrm{m} \mathrm{m}^{-2} \mathrm{~s}^{-1}\right)$, in a 1:1:1 mixture of sphagnum:perlite:vermiculite. To promote germination, seeds were stratified on soil at $4^{\circ} \mathrm{C}$ for 3 days in the dark. The Arabidopsis plants used in this work were in the Columbia-0 (Col-0) background. Mutant alleles and transgenic lines have been previously described: jaw- $D$ (Palatnik et al. 2003), tcp2-1 (SAIL_562_D05) (Schommer et al. 2008), nga1-4 nga2-3 nga3-3 nga4-1 and 35S::NGA3 (Trigueros et al. 2009).

\section{Sequence analyses}

NGA orthologs from rice, common bean and tomato were identified by standard BLAST searches, using NGA3 coding sequence (CDS) as a query. The flanking genomic sequences for these and for Arabidopsis NGA genes were then obtained from the database repositories of genome sequences for each species. Accession numbers of BACs containing the NGA genes are listed in Appendix S2, Supporting Information. Pairwise alignments of $1 \mathrm{~kb}$ genomic sequence for each gene upstream of the ATG codon were performed with the VISTA bioinformatic tool (Frazer et al. 2004). Following identification of conserved sequences, alignments of the most similar fragments were performed using the ClustalW tool in the Macvector 12.6 software.

\section{Y1H experiments}

The fragment used for the $\mathrm{Y} 1 \mathrm{H}$ screening was generated by annealing primers oPBF48 and oPBF50 (Appendix S1) and the subsequent synthesis of the reverse-complement strand using a Taq-mediated step of extension. The final product was cloned into pTUY1H (Castrillo et al. 2011), previously digested with $X b a l$ and $X$ mal restriction enzymes. For subsequent tests, a second bait construct was generated where a trimeric repetition of the 53 bp sequence used for the screening was cloned into the pTUY1H vector. The trimer was generated by two sequential polymerase chain reactions (PCRs) using in the first step the primers oPBF28 and OPBF41 and as a template the primer CFYtri; the resulting product was reamplified with primers oPBF46+oPBF47 and the final product was cloned into pCRII vector (Invitrogen, Carlsbad, CA), digested with Xbal and Xmal restriction enzymes and ligated into PTUY1H. See Appendix S1 for primer sequences. 
For the screening of the arrayed yeast library, transformation, growth and mating of yeast cells were performed as described previously (Castrillo et al. 2011, Rueda-Romero et al. 2012). The Saccharomyces cerevisiae $Y 187$ and $Y M 4271$ strains carrying bait and prey constructs, respectively, were used to test DNA-protein interactions. The screening was performed at $28^{\circ}$.

\section{Quantitative reverse transcription PCR}

Total RNA was extracted from A. thaliana Col-0, tcp2-1 and jaw-D seedlings with the E.Z.N.A. Plant RNA Kit (OMEGA Bio-Tek, Doraville, GA). Three micrograms of total RNA were used for cDNA synthesis with the First Strand CDNA Synthesis Kit (Invitrogen, Carslbad, CA), and the quantitative PCR master mix was prepared using the SYBR Premix Ex Taq (Takara Co., Tokyo, Japan). Primers used to amplify the NGA genes were as follows: OMNG48 and OMNG49 for NGA1, OMNG46 and OMNG47 for NGA2, OMNG42 and OMNG43 for NGA3 and OMNG44 and OMNG45 for NGA4. Results were normalized to the expression of the UBIQUITIN-CONJUGATING ENZYME21 (UBC21) mRNA, amplified with UBC21F and UBC21R primers. The PCR reactions were run and analyzed using the 7500 Fast Real-Time PCR System (Applied Biosystems, Foster City, CA). The Student's t-test was used to determine the significance of expression level differences. See Appendix S1 for primer sequences.

\section{GUS histochemical detection}

For $\beta$-glucuronidase (GUS) histochemical detection, samples were treated for $15 \mathrm{~min}$ in $90 \%$ ice-cold acetone and then washed for $5 \mathrm{~min}$ with washing buffer $(25 \mathrm{mM}$ sodium phosphate, $2 \mathrm{~m} M$ ferrocyanide, $2 \mathrm{mM}$ ferricyanide and $1 \%$ Triton $\mathrm{X}-100$ ) and incubated from 4 to $16 \mathrm{~h}$ at $37^{\circ} \mathrm{C}$ with staining buffer (washing buffer $+1 \mathrm{mM}$ $\mathrm{X}$-glucuronide). Following staining, plant material was fixed, cleared in chloral hydrate and mounted according to Weigel and Glazebrook (2002) to be viewed under bright-field microscopy. All detections were made in heterozygous lines for the reporter transgene.

To generate the NGA2::NGA2:GUS reporter, first the $3^{\prime}$ NGA2 genomic sequence downstream the STOP codon (+898 to +2919) was amplified with primers oMNG24 and oMNG25 and cloned into a pGREENII0029 vector (http://www.pgreen.ac.uk). The fragment of NGA2 genomic sequence spanning approximately $2.5 \mathrm{kp}$ upstream of the ATG and the NGA2 CDS $(-2575$ to +12$)$ was amplified with primers (oMNG22 and oMNG23) and subsequently cloned into a pCRII vector previously modified by introducing the GUS
CDS from pBI101.2. Finally, the fragment containing NGA2 upstream sequences, the NGA2 CDS and the GUS CDS, was digested from this intermediate plasmid and cloned into the pGREENII0029 vector already containing the 3 'genomic fragment downstream NGA2 CDS digested with Kpnl and Sall. See Appendix S1 for primer sequences.

\section{Genotyping}

Genotyping of nga mutants was performed as previously described (Trigueros et al. 2009). For tcp2-1 genotyping, guidelines from SIGNAL webpage were followed (http://signal.salk.edu). In brief, primers LP_SAIL_562_D05 and RP_SAIL_562_D05 were used to amplify the wild-type allele, while primers RP_SAIL_562_D05 and LB1 amplified the mutant allele. See Appendix S1 for primer sequences.

\section{Luciferase assays}

To generate promoter:LUC fusions for transient expression assays in Nicotiana benthamiana leaves, a 2.7-bp NGA3 promoter fragment $(-2722$ to ATG) was amplified from Arabidopsis Col-0 genomic DNA with primers A904-GUSd and oPBF11, cloned into pCRII vector (Invitrogen), digested with Hindlll and Ncol restriction enzymes and ligated into pGREEN_LUC (Hellens et al. 2005). To generate the NGA3 $2270:$ LuC reporter, NGA3 promoter fragments were amplified by a two-step sequential PCR. First, the fragments flanking the $270 \mathrm{bp}$ region were amplified using two primer combinations (A904-GUSd/oPBF53 and oPBF83/oPBF11) that produced two fragments with overlapping ends. An equimolecular mixture of both fragments was used as a template for a final PCR with primers A904-GUSd + oPBF11, and the final product was cloned into pCRII vector (Invitrogen), digested with HindIII and $\mathrm{Ncol}$ restriction enzymes and ligated into pGREEN_LUC (Hellens et al. 2005). Finally, the SINGAa promoter was amplified from Solanum lycopersicum CV. M82 genomic DNA with primers oPBF123 and oPBF124, cloned into pCRII vector (Invitrogen), digested with Smal and $\mathrm{Ncol}$ restriction enzymes and ligated into pGREEN_LUC (Hellens et al. 2005). The effector constructs were generated by amplifying the corresponding ORFs of TCP1, TCP2, TCP3 and $L A$ from CDNA of Arabidopsis Col-0 leaves (TCP1, TCP2 and TCP3) or $S$. lycopersicum cv Moneymaker leaves $(L A)$, that were subsequently cloned into PCR8/GW/TOPO (Invitrogen) and then transferred by Gateway reactions into the pMCD32 destination vector (TCP1, TCP2 and TCP3) (Curtis and Grossniklaus 2003) or the pEARLEY100 
destination vector (LA)(Earley et al. 2006). See Appendix $\mathrm{S} 1$ for primer sequences.

The transient expression assays were performed by Agrobacterium-infiltrated transient transformation of $N$. benthamiana, which was carried out as previously described (Espley et al. 2009). Briefly, N. benthamiana plants were grown until about $5 \mathrm{~cm}$ in height. Approximately, $300 \mu \mathrm{l}$ of Agrobacterium containing the reporter or/and effector plasmids were infiltrated into a young leaf at three points. Firefly luciferase and Renilla luciferase were assayed 3 days after infiltration using the Dual-Luciferase Reporter Assay System (Promega). Data were represented as the ratio of LUC/REN. Background controls were obtained by infiltrating only the reporter construct. At least three plants at the same developmental stage were used for each treatment, and the experiment was repeated three times. The Student's $t$-test was used to determine the significance of relative LUC activity differences.

\section{Results}

\section{TCP TF bind a conserved region in NGA promoters}

The four Arabidopsis NGA genes are expressed in highly similar domains, suggesting that they may share common regulators (Alvarez et al. 2009, Trigueros et al. 2009). To identify cis-regulatory elements that could mediate this regulation, we compared $1 \mathrm{~kb}$ of $5^{\prime}$ upstream promoter sequences of the four NGA genes by in silico analyses with the VISTA bioinformatic tool (Frazer et al. 2004). A region of approximately $270 \mathrm{bp}$ with significant similarity was identified around positions -300 to -30 upstream of the transcription start site in all NGA genes, where similarity was higher in NGA1/NGA2 and NGA3/NGA4 pairs. These regions contained a fragment of approximately $55 \mathrm{bp}$ in proximal positions that showed the highest level of conservation (Fig. 1). To identify putative TFs that could bind to these regions, we used a $55 \mathrm{bp}$ fragment of the NGA3 promoter spanning this highly conserved sequence to perform a $\mathrm{Y} 1 \mathrm{H}$ screening against an arrayed yeast library of ca. 1200 Arabidopsis TFs which has been previously described (Rueda-Romero et al. 2012). Seven positives were recovered from the screening, representing TFs from different families. Positive interactions were confirmed by two independent experiments using the same bait construct and a second construct where the $55 \mathrm{bp}$ sequence was cloned as a trimer upstream of the reporter genes to minimize false negatives by reinforcing positive DNA-TF interactions (Fig. 2). In these experiments, only three positives were unequivocally confirmed, all members of the class-II of the TCP family: TCP2 and TCP3, which belong
NGA1

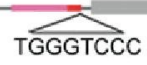

NGA2

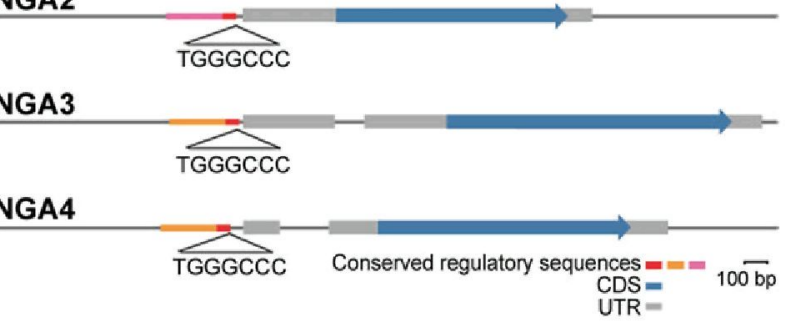

Fig. 1. Position of the conserved sequences in NGA promoters used in this study. A schematic representation of the four genomic regions for the Arabidopsis NGA genes is shown. The transcribed regions are noted with wide boxes where gray color corresponds to untranslated regions and blue color to coding sequences. Upstream of the transcription start, the position of the conserved sequences in the four promoters has been color-coded: red corresponds to the $55 \mathrm{bp}$ sequence showing highest similarity among the four promoters; pink corresponds to the fragment showing the highest similarity between the NGA1 and NGA2 promoters and orange to the fragment showing higher similarity between the NGA3 and NGA4 promoters. The regions represented in pink and orange are also similar to each other.

to the CIN-clade of the TCP family and are targets of the miR319; and TCP1, a member of the CYC/TB1 clade and not regulated by microRNA (miRNA) (Martín-Trillo and Cubas 2010).

\section{TCP2 and TCP3 are able to activate the NGA3 promoter}

To explore the functional relevance of TCP binding to the conserved sequence in NGA promoters, we tested the response of the NGA3 promoter in vivo to each of the three TCP factors identified in the screening. We used the Luc/Ren system described in Hellens et al. 2005. This system allows one to monitor the response of a given promoter to a TF in transient assays by measuring the activity of a $L U C I F E R A S E$ (LUC) reporter fused to the promoter under test. The construct also contains a Renilla (REN) gene under the control of the CaMv35S promoter to estimate the extent of the transient expression, which therefore serves as an internal standard when the activity of the promoter is represented as the ratio of LUC to REN activities (Luc/Ren) (Hellens et al. 2005). For our experiment, approximately $2.7 \mathrm{~kb}$ of $N G A 35^{\prime}$ genomic sequence ( -2722 to ATG) were cloned into the pGREENII 0800-Luc vector (NGA3::Luc). This promoter fragment was previously shown to recapitulate the native NGA3 expression in a NGA3::GUS reporter (Trigueros et al. 2009).

We expressed the NGA3::Luc construct in N. benthamiana leaves by agroinfiltration and found that in 


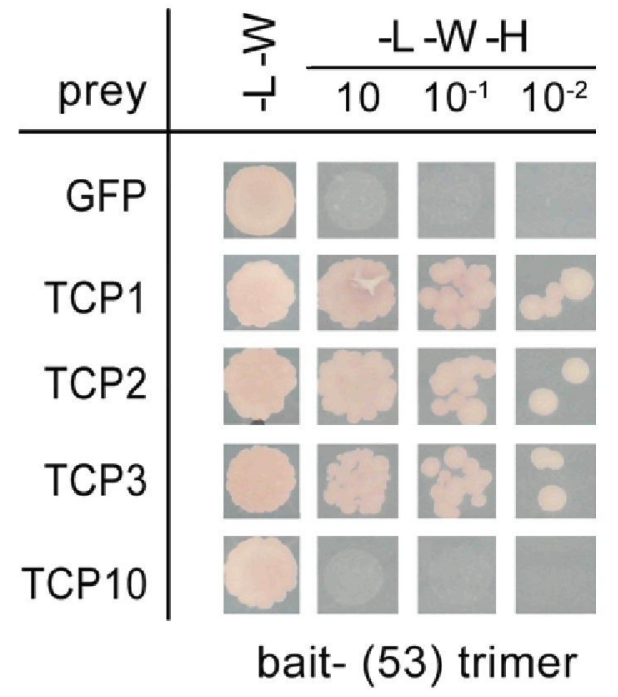

Fig. 2. TCP1, TCP2 and TCP3 bind to the $55 \mathrm{bp}$ conserved sequence in NGA promoters. A yeast strain containing a trimer repeat of the $53 \mathrm{bp}$ sequence used in the $\mathrm{Y} 1 \mathrm{H}$ screening cloned in the pTUY1H plasmid was mated to strains containing the activation domain (AD)-GFP (negative control), AD-TCP1, AD-TCP2, AD-TCP3 and AD-TCP10 constructs. Diploid cells were grown on non-selective minimal medium without Leu and $\operatorname{Trp}(-L-W)$ or selective minimal medium without Leu, Trp and His $(-L-W-H)$. Three serial dilutions of diploid cells were spotted. Only cells containing the TCP1, TCP2 and TCP3 factors were able to activate reporter expression, while the strains containing the negative control (GFP) or the related TCP10 factor were not able to grow in selective medium, supporting the specificity of the observed binding.

the absence of effectors, a significant basal level of LUC activity was observed, suggesting the presence of factors able to activate NGA3 transcription in the leaves of Nicotiana. Coinfiltration of the reporter with 35S::TCP2 or 35S::TCP3 produced upregulation of LUC activity (Fig. 2), whereas coinfiltration with 35S::TCP1 did not change the basal level of LUC activity conferred by the NGA3 promoter alone (Fig. 3). Next, we assayed a NGA3 $\triangle 270::$ Luc reporter where the $270 \mathrm{bp}$ fragment showing similarity among the four NGA promoters was eliminated. The NGA3 $\Delta 270$ promoter also conferred some basal level of activity, although much lower than the full NGA3 promoter, suggesting that this region was important for NGA3 regulation. We found that NGA3 $\triangle 270:$ :Luc activation in response to TCP2 or TCP3 was significantly reduced, but still higher that the basal level of LUC activity driven by the NGA3 $\Delta 270$ fragment (Fig. 3). These results strongly suggested that TCP2 and TCP3 could directly bind to this $270 \mathrm{bp}$ fragment to activate NGA expression in vivo, and that, likely, there were other redundant regions of the NGA3 promoter responsive to TCP2 and TCP3 outside this $270 \mathrm{bp}$ domain. Interestingly, a GGNCCC sequence that has been previously described as the TCP core-binding site

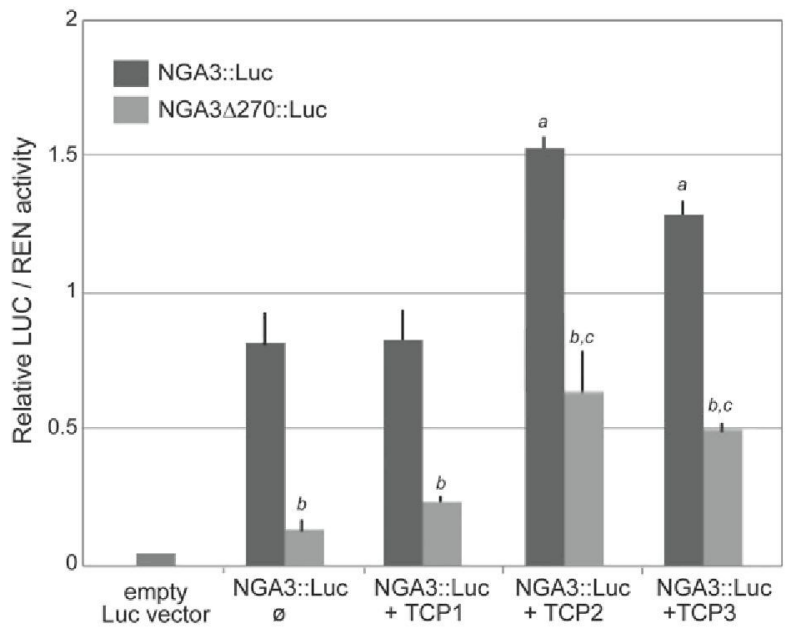

Fig. 3. Transient assays of NGA3::LUC expression. NGA3::LUC35S::REN and NGA3 $8270:: L U C-35 S::$ REN reporter constructs were transiently expressed in Nicotiana benthamiana leaves together with control vector, 355::TCP1, 355::TCP2 or 355::TCP3. The expression of REN was used as an internal control. Luc/Ren values represent means and standard errors $(n=6)$. Letters above bars indicate a significant difference according to Student's $t$-test $(P<0.05)$ from $(a)$ the values obtained when the NGA3::LUC-35S::REN reporter was infiltrated alone; (b) the values obtained when the full NGA3::LUC-35S::REN reporter was infiltrated with the corresponding effector and $(c)$ the values obtained when the NGA38270::LUC-35S::REN reporter was infiltrated alone.

was found in all four NGA promoters at the $3^{\prime}$ end of the 270 bp fragment (Fig. 1; Kosugi and Ohashi 2002).

\section{Expression of all NGA genes is reduced in tcp2 and jaw- $D$ mutants}

To test the functional relevance of the observed interactions between TCP factors and the NGA3 promoter in Arabidopsis, and whether they were also extensive to the rest of the NGA genes, the levels of all four NGA transcripts were quantified in $t c p 2$ single mutants. In this background, the expression of NGA2 and NGA3 was reduced, while NGA1 and NGA4 transcript levels were unchanged (Fig. 4A). Because a high degree of redundancy is found among CIN-like genes in Arabidopsis, $N G A$ expression was also determined in jaw- $D$ mutants, which overexpress MIR319a to simultaneously downregulate TCP2, TCP3, TCP4, TCP10 and TCP24 and show phenotypes similar to those caused by multiple combinations of loss-of-function alleles in these TCP genes (Palatnik et al. 2003, Koyama et al. 2010, Schommer et al. 2014). All NGA genes were expressed at significantly lower levels in jaw- $D$ mutants, indicating that TCP genes are required for $N G A$ regulation in a likely redundant manner (Fig. 4B)

To check if the effect in NGA regulation observed in jaw-D mutants affected not only the level but also 


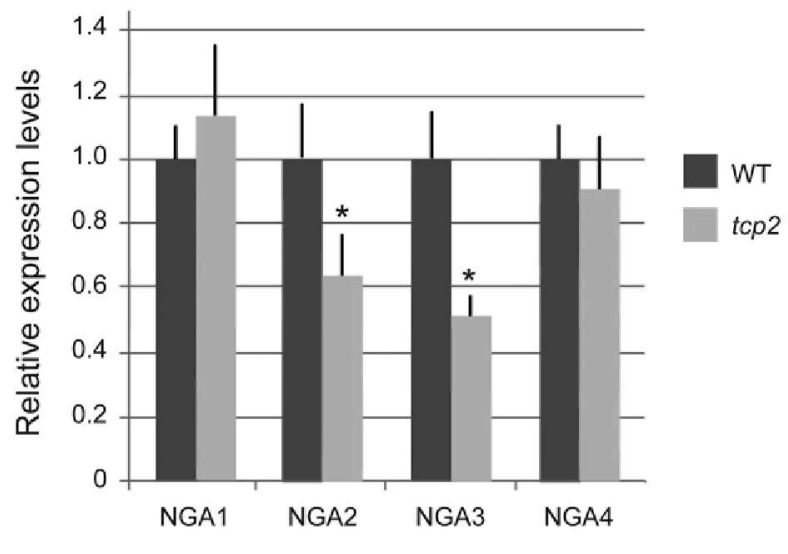

expression to optimal levels. However, because there is residual $T C P 2$ and $T C P 3$ expression in jaw-D mutants (Palatnik et al. 2003), it was not possible to rule out that these remaining levels of $T C P$ expression could be sufficient to activate NGA gene transcription

In summary, our data were consistent with TCP2 and TCP3 factors being true regulators of NGA expression in Arabidopsis, although mainly affecting expression levels and not spatial patterns.

\section{Leaf phenotypes associated with loss of function of $T C P$ genes that are targets of miR319a could be partially caused by reduced $N G A$ expression}

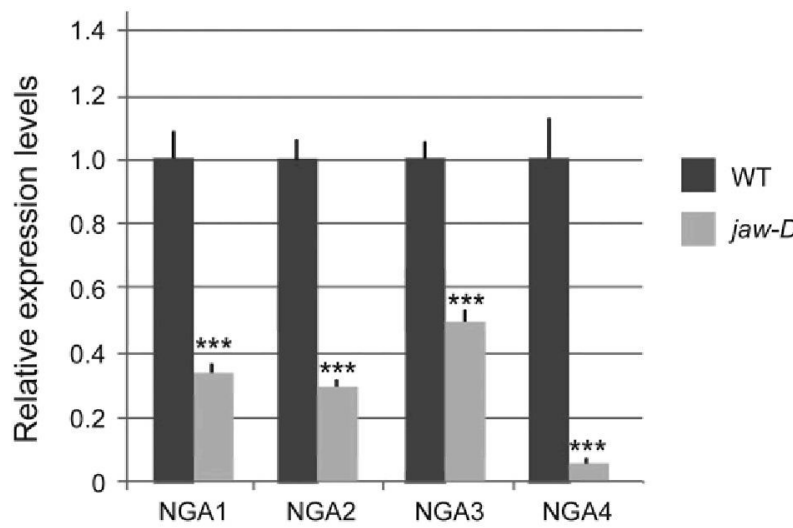

Loss of function of miR319a-target TCP genes of the CIN subfamily causes defects in leaf development. Thus, leaves of $t c p 2$, tcp 4 or tcp 10 single mutants are wider and slightly more serrated than wild type (Schommer et al. 2008), and when combined, the severity of the phenotype is strongly enhanced, as it also occurs in jaw-D mutants (Palatnik et al. 2003, Schommer et al. 2008, Koyama et al. 2010), Fig. 6A, C-D, G-J). These phenotypes partly resemble those found in nga quadruple mutants, where leaves are also wider and more serrated than wild type (Alvarez et al. 2009, Trigueros et al. 2009), Fig. 6B, K). Because NGA genes appear to be positively regulated by TCP2 and TCP3, it could be possible that the tcp2 or $t c p 3$ phenotypes were due to the weakly reduced levels of NGA expression in these mutants. If this was true, overexpression of NGA genes in these backgrounds should complement the observed tcp leaf phenotypes. 35S::NGA3 tcp2 plants showed phenotypes indistinguishable from those of 355 ::NGA3 lines, supporting this hypothesis (Fig. $6 \mathrm{~N}$ ). We also tested the effect of NGA3 overexpression in the jaw-D background, where the expression of all NGA genes is strongly reduced. Because jaw-D and 35S::NGA3 confer dominant phenotypes, we compared leaf morphology in the F1 plants (hemizygous to $355:: N G A 3$ and heterozygous for $j a w-D$ allele) to heterozygous $j a w-D$ and hemizygous $35 \mathrm{~S}:$ NGA3 plants. Rosette leaves of 35S::NGA3 jaw-D plants were much narrower than the corresponding heterozygous jaw- $D$ leaves, indicating that NGA3 overexpression was able to significantly suppress lateral growth produced by loss of TCP function. Leaf serration was also reduced by NGA3 overexpression, although it still was more conspicuous than in wild type or in 35S::NGA3 lines (Fig. 6C-F, H-I, L-M). signal appeared to be weaker in jaw-D mutants (Fig. 5). These results indicated that TCP regulation of NGA genes mainly affected the levels of $N G A$ gene expression, but not the spatial distribution of NGA transcripts, and that CIN-like TCP genes were not required for the onset of NGA expression, but were important to maintain NGA

\section{The TCP/NGA regulatory module appears to be conserved in crop species}

$T C P$ genes have been shown to control leaf morphology in several species, including tomato, rice and legumes. 

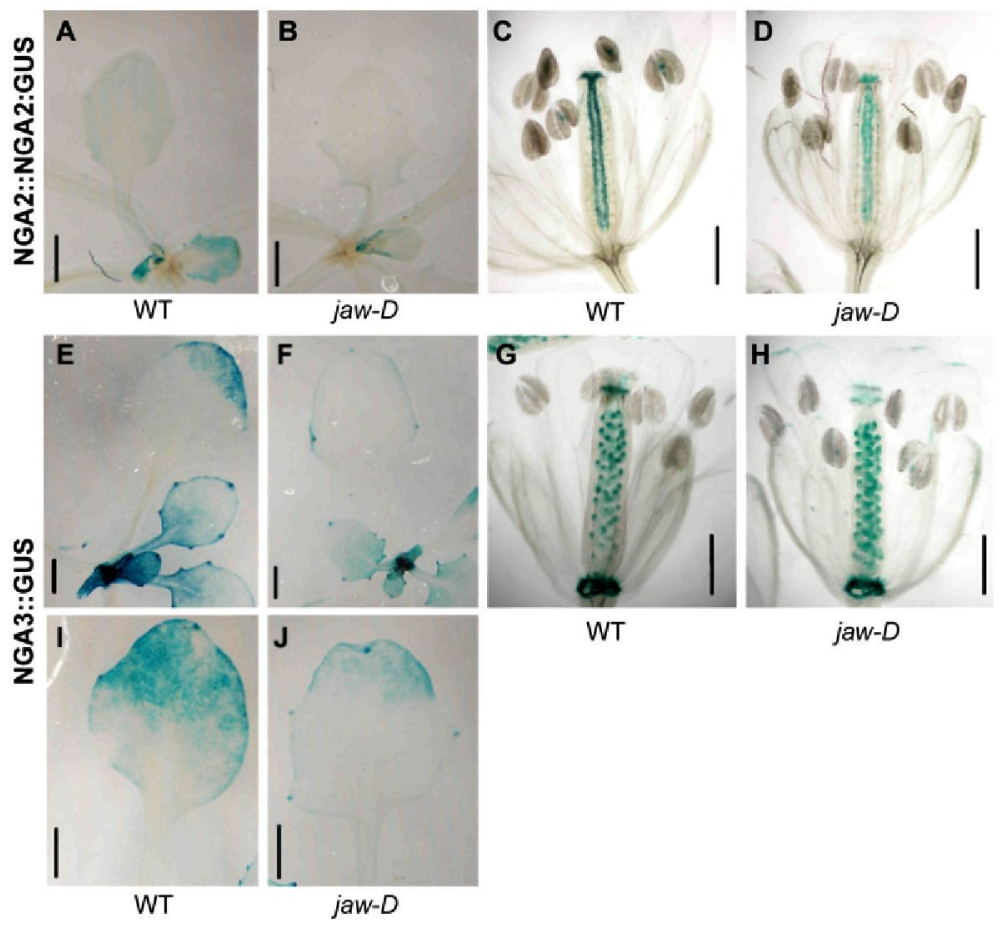

Fig. 5. The effect of jaw-D mutation in the activity of NGA2 and NGA3 reporters. NGA2::NGA2: GUS expression in seedlings of (A) wild type or (B) jaw-D/+ mutant plants and in anthesis flowers of (C) wild type or (D) jaw-D/+ mutants. NGA3::GUS expression in seedlings of (E) wild type or (F) jaw-D/+ mutant plants and in anthesis flowers of $(G)$ wild type or $(H)$ jaw-D/+ mutants. Detail of NGA3::GUS reporter activity in a young wild-type leaf $(\mathrm{I})$ and a jaw-D/+ leaf at an equivalent stage $(\mathrm{J})$. In all cases, the expression patterns are similar in wild type or jaw-D/+ backgrounds, although GUS activity is detected at lower levels in jaw-D/+ mutants. Bar $=1 \mathrm{~mm}$ in $\mathrm{A}, \mathrm{B}, \mathrm{E}, \mathrm{F}, \mathrm{I}$ and J. Bar $=500 \mu \mathrm{m}$ in $\mathrm{C}, \mathrm{D}, \mathrm{G}$ and $\mathrm{H}$.

The conservation of NGA function across angiosperms has not been so extensively explored, although it has been shown that, at least in eudicots, NGA factors have an essential role in style and stigma development in distantly related species. Moreover, N. benthamiana flowers with reduced NGA activity showed altered sepal and petal development, suggesting that NGA role in lateral organ expansion is also conserved (Fourquin and Ferrandiz 2014). To test whether the observed interaction between TCP factors and NGA promoters was part of a conserved regulatory module in other species, we compared the promoters of NGA orthologs from Arabidopsis, rice, tomato and common bean. We found that the 53 bp core sequence detected in Arabidopsis NGA promoters was also present in several NGA orthologs in these species, including the putative TCP-binding site, and strongly suggesting that NGA genes could be regulated by TCP factors in these species as well (Fig. 7A).

To validate in vivo this suggested interaction, we used again the Luc/Ren system to test whether LANCEOLATE (LA) [the tomato ortholog of AtTCP3 (Ori et al. 2007)] was able to activate the promoter of SINGAa (the tomato ortholog of AtNGA3). A fragment of approximately $2 \mathrm{~kb}$ of the SINGAa promoter was cloned into the
pGREEN-Luc 800 vector. Coinfiltration of the SINGAa reporter with $35 \mathrm{~S}:$ :LA resulted in a significant increase of the Luc/Ren ratio, indicating that LA was able to activate the SINGAa promoter in planta (Fig. 7B). Likewise, LA could also activate the Arabidopsis NGA3::Luc reporter to a similar extent as its Arabidopsis ortholog, AtTCP3 (Fig. 7C). Altogether, these results supported the hypothesis of the conservation of a TCP-NGA regulatory module in distantly related species.

\section{Discussion}

In this work, we have found that TCP1, TCP2 and TCP3 are able to bind a fragment of the NGA3 promoter in a $\mathrm{Y} 1 \mathrm{H}$ experiment, whose sequence is highly conserved within the promoters of the four NGA genes. In addition, we show that TCP2 and TCP3, but not TCP1, are able to activate the transcription of a reporter gene fused to the NGA3 promoter in planta. Moreover, the expression levels of all NGA genes are strongly reduced in the jaw- $D$ background, where TCP2, TCP3 and other $\mathrm{CIN}$-like $T C P$ genes are downregulated. The potential role of $\mathrm{CIN}$-like TCP factors in the upregulation of NGA genes had been already suggested by a previous work that showed 


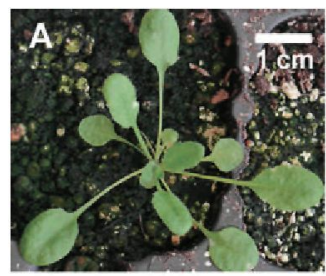

WT

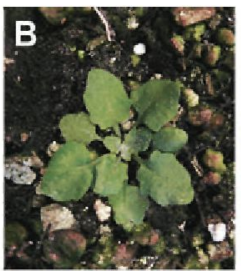

nga $1 / 2 / 3 / 4$

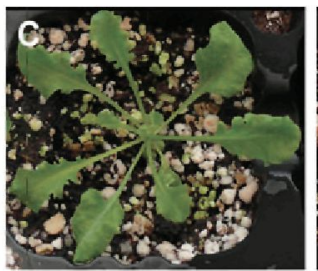

jaw- $D$

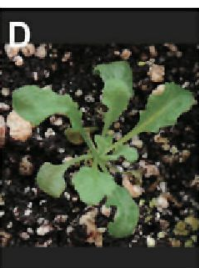

jaw-D/+

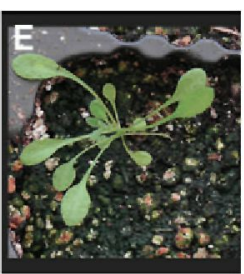

35S::NGA3/+

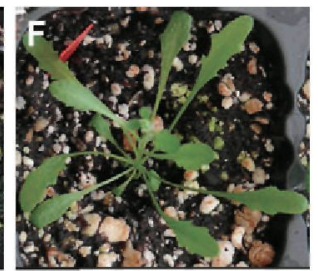

jaw-D/+ 35S::NGA3/+

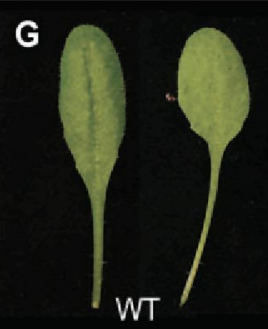

H

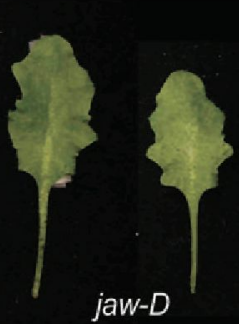

I
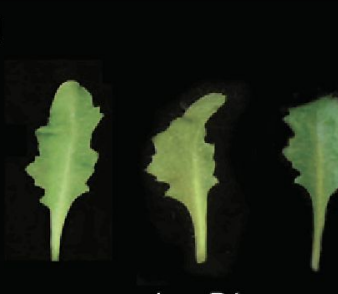

jaw-D/+
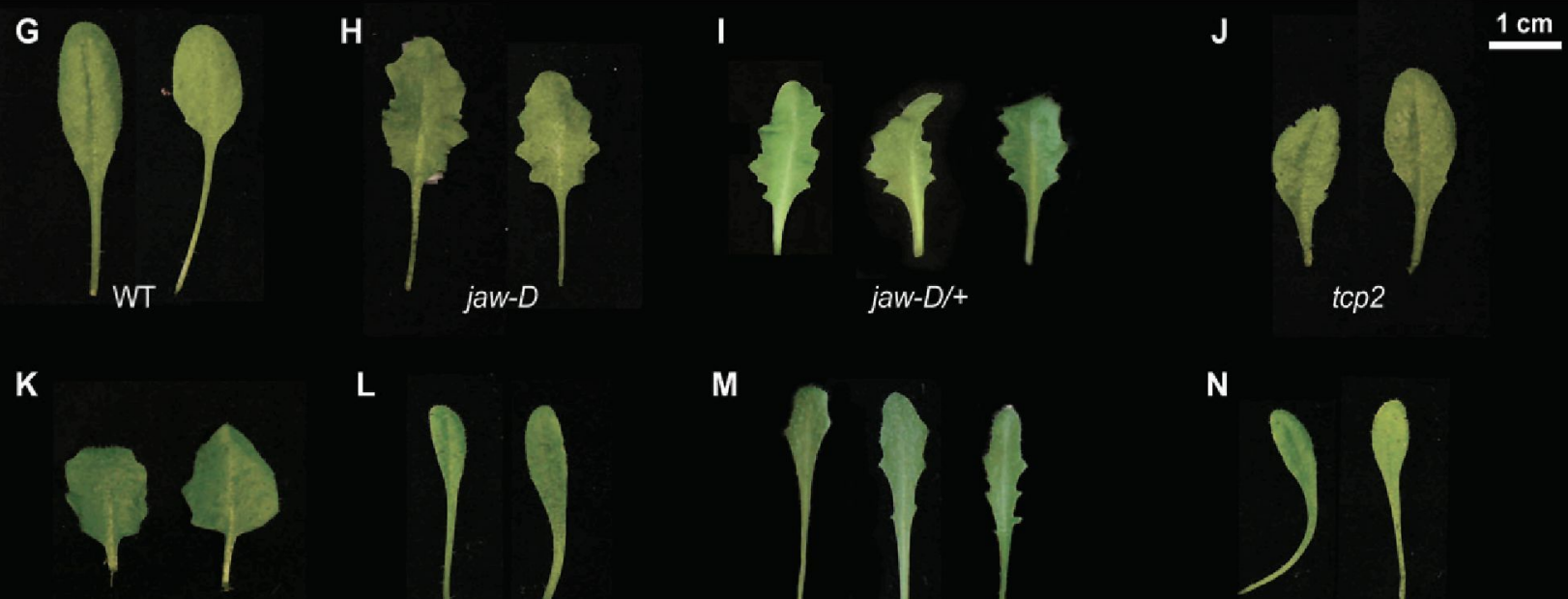

L

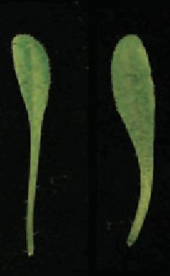

M

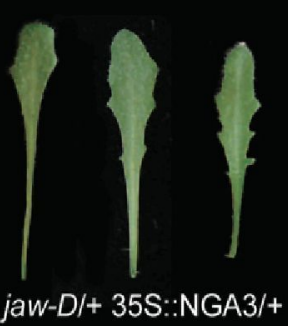

N

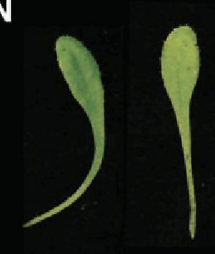

jaw-D/+35S: NGA3/+

tcp2 35S::NGA3/+

Fig. 6. Phenotypes of the different mutants and combinations generated. Rosettes of (A) wild type, (B) nga1 nga2 nga3 nga4 quadruple mutant, (C) jaw-D homozygous mutant, (D) jaw-D heterozygous mutant, (E) 35S::NGA3 hemizygous line, (F) an F1 plant derived from the cross of jaw-D and 35S::NGA3, therefore heterozygous for jaw-D and hemizygous for 35S::NGA3. Dissected leaves from plants of different genotypes at equivalent stages: (G) wild type. (H) jaw-D homozygous. (I) jaw-D heterozygous. (J) tcp2 mutant. (K) nga1 nga2 nga3 nga4 quadruple mutant. (L) $355:$ :NGA3 hemizygous. (M) jaw-D heterozygous, 35S: :NGA hemizygous. (N) tcp2 35S::NGA3 hemizygous. Bars $=1 \mathrm{~cm}$.

reduced levels of NGA3 and NGA4 expression upon estradiol-mediated induction of a ProXVE::TCP3:SRDX transgene (Koyama et al. 2010). Our results, which identify TCP factors as putative regulators of NGA genes through a completely different approach, provide further functional data that confirm TCP2 and TCP3 as likely direct activators of NGA gene expression.

CIN-like TCP clade in Arabidopsis comprise five genes regulated by miR319 (TCP2, TCP3, TCP4, TCP10 and $T C P 24)$ and three other genes not regulated by miRNAs (TCP5, TCP13 and TCP17) (Martín-Trillo and Cubas 2010). These eight genes act in a partially redundant manner to control several aspects of leaf development, as deduced from the mild phenotypes of single mutants in several of these genes and the increasing severity of phenotypic alterations seen in multiple combinations of the different mutants or when simultaneous downregulation of the eight genes is achieved by overexpression of miR319 and an artificial miRNA targeting TCP5, TCP13 and TCP17 (Efroni et al. 2008, Schommer et al. 2008, Koyama et al. 2010). The functional relevance of the regulatory TCP-NGA interaction uncovered by this work is well supported by the phenotypes of nga quadruple mutants, which show enlarged serrated leaves resembling low order mutant combinations in CIN-like TCP genes, but are much less severe than those of high order $c i n-t c p$ mutant combinations. Thus, nga leaf phenotypes are a subset of those caused by complete loss of CIN-like TCP function. Likewise, NGA overexpression results in phenotypes resembling those caused by overexpression of miR319-resistant versions of $T C P 2, T C P 3$ or $T C P 4$, which include narrow epinastic leaves, fused cotyledons and smooth leaf margins (Palatnik et al. 2003, Koyama et al. 2007, Trigueros et al. 2009). Moreover, 35S::NGA3 tcp2 plants were indistinguishable from $355:$ :NGA3 lines, and overexpression of $N G A 3$ in a jaw-D background suppressed part of the phenotypes associated with miR319 overexpression, especially the lateral growth of the lamina and, to some extent, the exaggerated leaf serration (Fig. 6). Altogether, this genetic evidence suggests that NGA genes act downstream of TCP factors to partially mediate their functions. 
A

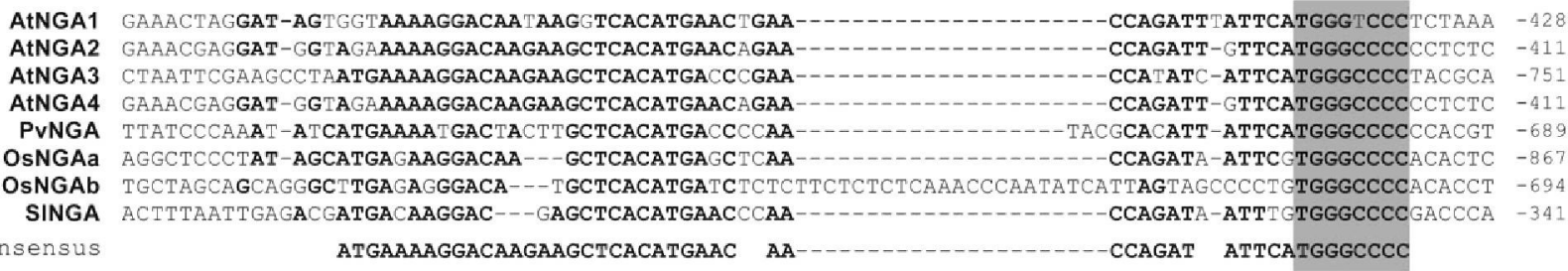

TCP core binding seq

B

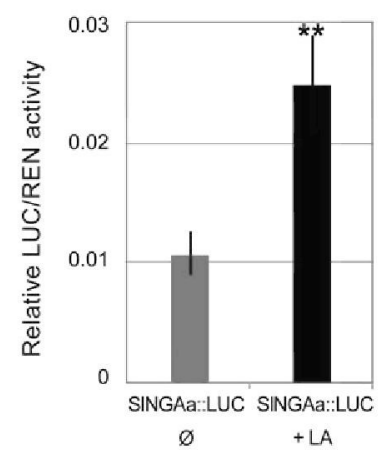

C

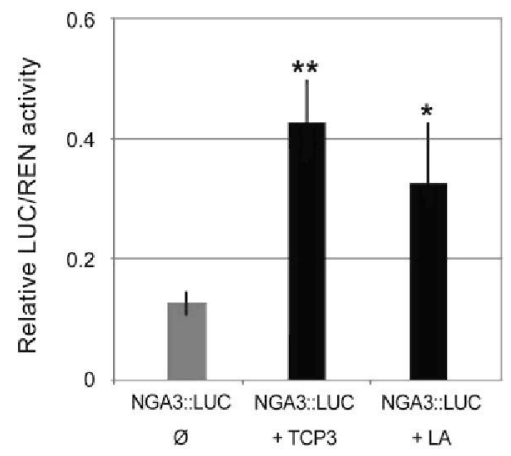

Fig. 7. Conservation of NGA promoter sequence and activity in different species. (A) Alignment of a fragment of NGA promoters from different species showing similarity to the 55 bp sequence in AtNGA3 promoter used in the $Y 1 \mathrm{H}$ screening. The putative TCP-binding site is shadowed. In bold, most conserved bases are shown. Numbers on the right correspond to the relative position of the last base to the START codon of the corresponding gene. Accession numbers are as follows: AtNGA1 (At2g46870), AtNGA2 (At3g61970), AtNGA3 (At1g01030), AtNGA4 (At4g01500), PVNGA (PHAVU_010G120900g), OsNGAa (LOC_Os03g02900), OsNGAb (LOC_Os10g39190), SINGAa (LOC101249569). (B) Transient assays of SINGAa::LUC expression. SINGAa::LUC-35S::REN reporter construct was transiently expressed in Nicotiana benthamiana leaves together with control vector or 35S::LANCEOLATE (35S::LA). The expression of REN was used as an internal control. Luc/Ren values represent means and standard errors $(n=6)$. Asterisks indicate a significant difference from the values obtained when the SINGAa::LUC-35S::REN reporter was infiltrated alone according to Student's $t$-test ( $* * P<0.01$ ). (C) Transient assays of NGA3::LUC expression in response to the heterologous TCP factor from tomato LA. NGA3::LUC-35S::REN reporter construct was transiently expressed in $N$. benthamiana leaves together with control vector, $355:$ TCP3 (the ortholog of LA in Arabidopsis) and 35S::LA. The expression of REN was used as an internal control. Luc/Ren values represent means and standard errors ( $n=6$ ). Asterisks indicate a significant difference from the values obtained when the NGA3::LUC-35S::REN reporter was infiltrated alone according to Student's t-test $\left({ }^{*} P<0.05 ; * *, P<0.01\right)$.

This is compatible with different and not mutually exclusive scenarios:

1. First, CIN-like TCP factors participate in multiple regulatory interactions and developmental processes that could be genetically separated. For example, they have been shown to regulate leaf shape through negative regulation of boundary genes, namely the CUP-SHAPED COTYLEDON (CUC) genes. This negative regulation is achieved by parallel convergent pathways, which involve the independent activation by TCP of different factors such as ASSYMETRIC LEAVES1, miR164 or auxin-related genes, that in turn repress CUC expression (Koyama et al. 2007, Koyama et al. 2010). In addition, CIN-like TCP factors also control leaf development through CUC-independent mechanisms such as the control of cell proliferation through activation of miR396,
CYCLIN-DEPENDENT KINASE INHIBITOR/KIP RELATED PROTEIN1 and jasmonate biosynthesis (Schommer et al. 2014), or interfering with cytokinin signaling (Efroni et al. 2013). In this context, we can envision that NGA genes would be involved in only part of these multiple pathways, therefore only contributing to a subset of TCP functions. In fact, CIN-like TCP and NGA factors have been related to common pathways like auxin signaling (Trigueros et al. 2009, Martinez-Fernandez et al. 2014), cytokinin response (Kwon et al. 2011) or control of cell proliferation (Kwon et al. 2009), and detailed genetic analyses will have to be performed to elucidate the NGA-dependent and NGA-independent modes of TCP function.

2 . The weaker phenotype on nga quadruple mutants compared with multiple cin-tcp mutants could be due to redundancy of NGA genes with the 
related three NGA-LIKE (NGAL) genes also present in the Arabidopsis genome (At3g11580, At5g06250 and At2g36080) (Alvarez et al. 2009). Interestingly, it has been recently reported that DEVELOPMENT-RELATED PCG TARGET IN THE APEX 4, which corresponds to NGAL2, controls leaf serration in Arabidopsis by negatively regulating CUC2 (Engelhorn et al. 2012). Likewise, it has been described that the overexpression of any of the NGAL genes also causes suppression of leaf margin serration and inhibition of lateral growth of the lamina (Alvarez et al. 2009, Engelhorn et al. 2012, Shao et al. 2012). Thus, it seems likely that NGA and NGAL factors share redundant functions in leaf development, which should be unveiled by generating multiple mutant combinations in all seven loci that have not been achieved so far. These multiple mutants could also be used to compare the effects of full loss of NGANGAL function and of CIN-TCP function to understand further the extent of their functional relationship.

3 . It could be possible that NGA genes are only activated by a subset of TCP factors, and therefore only mediate the function of these in leaf development. Thus, nga phenotypes could reflect the specific contribution of this TCP subset to the process. In fact, the $\mathrm{Y} 1 \mathrm{H}$ screening performed in this work only identified three TCP factors as able to bind to the $55 \mathrm{bp}$ conserved fragment in NGA promoters, even though all members of the TCP family except TCP7 were present in the arrayed collection used for the screening. While we cannot exclude that we obtained some false negatives, these results suggest that not all CIN-TCP factors regulate NGA expression. In fact, it has been clearly shown that not all CIN-like TCP factors are fully equivalent: there are clear differences among the expression patterns of the different CIN-like TCP genes (Koyama et al. 2007, Danisman et al. 2013), their protein-protein interactions (Danisman et al. 2013) or the severity of the phenotypes caused by their overexpression (Palatnik et al. 2003, Koyama et al. 2007, Danisman et al. 2013, Li and Zachgo 2013), so it is conceivable that there are some constrains to the observed TCP-NGA regulatory interactions based on these differences. Still, we cannot completely rule out that other TCP factors in addition to TCP2 and TCP3 regulate NGA expression. Our transient expression analyses of TCP effect on the NGA3 promoter suggest that not only TCP2/TCP3 activation of NGA expression is mediated by the conserved region used in the $\mathrm{Y} 1 \mathrm{H}$ screening but that other TCP-responsive regulatory elements are also present in different positions of the NGA3 promoter. To test whether these additional elements could also respond to other members of the CIN-like TCP clade, it would be interesting to pursue a systematic study in which all TCP factors should be tested. These experiments could also add some functional evidence to clarify the differences among related TCP factors that act redundantly but are not fully equivalent

In addition to their general role in lateral organ development, NGA factors are essential to specify the identity of the apical tissues in the gynoecium, the style and the stigma. According to publicly available microarray data, TCP2 and TCP3 are expressed in carpels (Atgenexpress, http://www.arabidopsis.org/portals/expression/ microarray/ATGenExpress.jsp), and we observed reduced NGA levels in jaw-D gynoecia. However, jaw- $D$ mutants only show mild defects in fruit development that mainly affect fruit elongation, but not style or stigma development (Palatnik et al. 2003), suggesting that the NGA role in apical gynoecium patterning does not significantly depend on TCP activity.

Finally, our work points to the likely conservation of the uncovered TCP-NGA regulatory module to control leaf development across angiosperm species. We were able to find the highly conserved sequences identified in the four Arabidopsis NGA promoters in NGA ortholog genes from distant crop species such as rice (a monocot), tomato (Solanaceae) or common bean (legume). Moreover, we show that one of these promoters (SINGAa) was also activated by the corresponding tomato TCP3 ortholog in a transient expression assay. Previous reports have shown that CIN-TCP genes control leaf development in several species from different clades within angiosperms. Thus, the study of the cincinnata mutants in Antirhinum majus, which show severely crinkled leaves, led to the identification of the first CIN-TCP gene (Nath et al. 2003); in tomato, a gain-of-function mutation in the $L A$ gene causes a extreme reduction of leaf complexity and size, while overexpression of miR319 results in the increase of leaflet size and the formation of crinkled leaf margins (Ori et al. 2007); likewise, overexpression of miR319 causes leaf blade expansion in rice or in creeping bentgrass (Yang et al. 2013, Zhou et al. 2013). The well-established general role of CIN-TCP genes in leaf development across angiosperms is in contrast with the almost non-existent knowledge about the role of NGA genes in leaf development in other species than Arabidopsis. However, a recent study shows that NGA genes play similar roles in gynoecium development in distantly related species within eudicots and that, in N. benthamiana, other lateral organs such as petals or sepals are 
affected by NGA downregulation (Fourquin and Ferrandiz 2014), suggesting that the NGA function in controlling lateral organ growth could also be conserved. In this work, we were able to uncover a likely conserved regulatory interaction of TCP and NGA factors in different species based on the comparison of promoter sequences and transient expression experiments. Even though the functional relevance of this interaction remains to be tested, a simple strategy has allowed us to propose the existence of a regulatory module that could be important to explain leaf development in crop species and therefore justify further work that could provide novel insights in basic questions on leaf morphogenesis, as well as identify new targets for biotechnological crop improvement.

\section{A contributions}

P. B. designed and performed most of the experiments, together with M. N. G. P.C. and L.O.S. contributed the $\mathrm{Y} 1 \mathrm{H}$ system and helped with these experiments. C. F conceived the idea, designed most of the experiments, supervised the work and wrote the manuscript.

Acknowledgements-This work was supported by the Spanish Ministerio de Ciencia e Innovación (grants BlO2009-09920 to C.F. and BlO2010-17334 to L.O.) and the Spanish Ministerio de Economia y Competitividad (grants BIO2012-32902 to C. F. and BIO2013-46076-R to L.O.) and Predoctoral Fellowships to P.B. (JAE-Pre, from CSIC) and M. N.G (Generalitat Valenciana). Furthermore, C. F. acknowledges the support of the European Union FP7 project EVOCODE (grant 247587). We thank Irene Martínez-Fernández, Francisco Madueño, Concha Gomez Mena, Amelia Felipo and Laura Campos (IBMCP) for providing material and technical advice and Antonio Villar Orozco (IBMCP) for technical assistance in the greenhouse.

\section{$\mathbf{R}$}

Alvarez JP, Goldshmidt A, Efroni I, Bowman JL, Eshed Y (2009) The NGATHA distal organ development genes are essential for style specification in Arabidopsis. Plant Cell 21: 1373-1393

Araki T, Komeda Y (1993) Analysis of the role of the late-flowering locus, $\mathrm{Gl}$, in the flowering of Arabidopsis thaliana. Plant J 3: 231-239

Bleecker AB (1998) The evolutionary basis of leaf senescence: method to the madness? Curr Opin Plant Biol 1: 73-78

Castrillo $\mathrm{G}$, Turck F, Leveugle $M$, Lecharny A, Carbonero P, Coupland G, Paz-Ares J, Onate-Sanchez L (2011) Speeding cis-trans regulation discovery by phylogenomic analyses coupled with screenings of an arrayed library of Arabidopsis transcription factors. PLoS One 6: e21524

Curtis MD, Grossniklaus U (2003) A gateway cloning vector set for high-throughput functional analysis of genes in planta. Plant Physiol 133: 462-469

Danisman S, van Dijk AD, Bimbo A, van der Wal F, Hennig L, de Folter S, Angenent GC, Immink RG (2013) Analysis of functional redundancies within the Arabidopsis TCP transcription factor family. J Exp Bot 64: $5673-5685$

Earley KW, Haag JR, Pontes O, Opper K, Juehne T, Song K, Pikaard CS (2006) Gateway-compatible vectors for plant functional genomics and proteomics. Plant J 45: 616-629

Efroni I, Blum E, Goldshmidt A, Eshed Y (2008) A protracted and dynamic maturation schedule underlies Arabidopsis leaf development. Plant Cell 20: 2293-2306

Efroni I, Han SK, Kim HJ, Wu MF, Steiner E, Birnbaum KD, Hong JC, Eshed Y, Wagner D (2013) Regulation of leaf maturation by chromatin-mediated modulation of cytokinin responses. Dev Cell 24: 438-445

Engelhorn J, Reimer IJ, Leuz I, Gobel U, Huettel B, Farrona S, Turck F (2012) Development-related PcG target in the apex 4 controls leaf margin architecture in Arabidopsis thaliana. Development 139: 2566-2575

Espley RV, Brendolise C, Chagne D, Kutty-Amma S, Green S, Volz R, Putterill J, Schouten HJ, Gardiner SE, Hellens RP, Allan AC (2009) Multiple repeats of a promoter segment causes transcription factor autoregulation in red apples. Plant Cell 21: 168-183

Fourquin C, Ferrandiz C (2014) The essential role of NGATHA genes in style and stigma specification is widely conserved across eudicots. New Phytol 202: $1001-1013$

Frazer KA, Pachter L, Poliakov A, Rubin EM, Dubchak I (2004) VISTA: computational tools for comparative genomics. Nucleic Acids Res 32: W273-W279

Hellens RP, Allan AC, Friel EN, Bolitho K, Grafton K, Templeton MD, Karunairetnam S, Gleave AP, Laing WA (2005) Transient expression vectors for functional genomics, quantification of promoter activity and RNA silencing in plants. Plant Methods 1: 13

Koenig D, Sinha N (2010) Evolution of leaf shape: a pattern emerges. Curr Top Dev Biol 91: 169-183

Kosugi S, Ohashi Y (2002) DNA binding and dimerization specificity and potential targets for the TCP protein family. Plant J 30: 337-348

Koyama T, Furutani M, Tasaka M, Ohme-Takagi M (2007) TCP transcription factors control the morphology of shoot lateral organs via negative regulation of the expression of boundary-specific genes in Arabidopsis. Plant Cell 19: 473-484

Koyama T, Mitsuda N, Seki M, Shinozaki K, Ohme-Takagi $M(2010)$ TCP transcription factors regulate the activities of ASYMMETRIC LEAVES1 and miR164, as well as the 
auxin response, during differentiation of leaves in Arabidopsis. Plant Cell 22: 3574-3588

Kwon SH, Chang SC, Ko JH, Song JT, Kim JH (2011) Overexpression of Brassica rapa NGATHA1 gene confers de-etiolation phenotype and cytokinin resistance on Arabidopsis thaliana. J Plant Biol 54: 119-125

Kwon SH, Lee BH, Kim EY, Seo YS, Lee S, Kim WT, Song JT, Kim JH (2009) Overexpression of a Brassica rapa NGATHA gene in Arabidopsis thaliana negatively affects cell proliferation during lateral organ and root growth. Plant Cell Physiol 50: 2162-2173

Li S, Zachgo S (2013) TCP3 interacts with R2R3-MYB proteins, promotes flavonoid biosynthesis and negatively regulates the auxin response in Arabidopsis thaliana. Plant J 76: $901-913$

Martín-Trillo M, Cubas P (2010) TCP genes: a family snapshot ten years later. Trends Plant Sci 15: $31-39$

Martinez-Fernandez I, Sanchis S, Marini N, Balanza V, Ballester P, Navarrete-Gomez M, Oliveira AC, Colombo L, Ferrandiz C (2014) The effect of NGATHA altered activity on auxin signaling pathways within the Arabidopsis gynoecium. Front Plant Sci 5: 210

Nath U, Crawford BCW, Carpenter R, Coen E (2003) Genetic control of surface curvature. Science 299 : 1404-1407

Ori N, Cohen AR, Etzioni A, Brand A, Yanai O, Shleizer S, Menda N, Amsellem Z, Efroni I, Pekker I, Alvarez JP, Blum E, Zamir D, Eshed Y (2007) Regulation of LANCEOLATE by miR319 is required for compound-leaf development in tomato. Nat Genet 39: 787-791

Ostergaard L (2009) Don't 'leaf' now. The making of a fruit. Curr Opin Plant Biol 12: 36-41

Palatnik JF, Allen E, Wu X, Schommer C, Schwab R, Carrington JC, Weigel D (2003) Control of leaf morphogenesis by microRNAs. Nature 425: 257-263

Rueda-Romero P, Barrero-Sicilia C, Gomez-Cadenas A, Carbonero P, Onate-Sanchez L (2012) Arabidopsis thaliana DOF6 negatively affects germination in non-after-ripened seeds and interacts with TCP14. J Exp Bot 63: 1937-1949

Schommer C, Debernardi JM, Bresso EG, Rodriguez RE, Palatnik JF (2014) Repression of cell proliferation by miR319-regulated TCP4. Mol Plant 7: 1533-1544
Schommer C, Palatnik JF, Aggarwal P, Chetelat A, Cubas P, Farmer EE, Nath U, Weigel D (2008) Control of jasmonate biosynthesis and senescence by miR319 targets. PLoS Biol 6: e230

Shao J, Liu X, Wang R, Zhang G, Yu F (2012) The over-expression of an Arabidopsis B3 transcription factor, ABS2/NGAL1, leads to the loss of flower petals. PLoS One 7: e49861

Sundberg E, Ferrándiz C (2009) Gynoecium patterning in Arabidopsis: a basic plan behind a complex structure. In: Annual Plant Reviews Volume 38: Fruit Development and Seed Dispersal, (ed L. Ostergaard), Wiley-Blackwell, Oxford, UK, pp 35-69

Sundberg E, Ostergaard L (2009) Distinct and dynamic auxin activities during reproductive development. Cold Spring Harb Perspect Biol 1: a001628

Trigueros M, Navarrete-Gómez M, Sato S, Christensen SK, Pelaz S, Weigel D, Yanofsky MF, Ferrándiz C (2009) The NGATHA genes direct style development in the Arabidopsis gynoecium. Plant Cell 21: 1394-1409

Weigel D, Glazebrook J (2002). Arabidopsis: A Laboratory Manual (Cold Spring Harbor, NY: Cold Spring Harbor Laboratory Press)

Yang C, Li D, Mao D, Liu X, Ji C, Li X, Zhao X, Cheng Z, Chen C, Zhu L (2013) Overexpression of microRNA319 impacts leaf morphogenesis and leads to enhanced cold tolerance in rice (Oryza sativa L.). Plant Cell Environ 36: $2207-2218$

Zhou M, Li D, Li Z, Hu Q, Yang C, Zhu L, Luo H (2013) Constitutive expression of a miR3 19 gene alters plant development and enhances salt and drought tolerance in transgenic creeping bentgrass. Plant Physiol 161 1375-1391 